\title{
Tactile Perception of Rotational Sliding
}

\author{
William R. Provancher and Brandt D. Erickson \\ University of Utah \\ Haptics and Embedded Mechatronics Lab \\ Salt Lake City, Utah \\ $\{$ wil, berickso\}@eng.utah.edu
}

\author{
Federico Barbagli \\ Stanford University \\ Stanford Robotics Lab \\ Stanford, California \\ barbagli@stanford.edu
}

\author{
Hong Z. Tan \\ Purdue University \\ Haptic Interface Research Lab \\ West Lafayette, Indiana \\ hongtan@purdue.edu
}

\begin{abstract}
We present a new tactile display for rendering rotational friction at the fingertips. To inform the design and control of this device, we have conducted an initial set of perceptual experiments to investigate perceptual thresholds for rates of rotational slipping at the fingertips. It was found that humans can discriminate a change in rotational sliding rate of 4.13-28.04 rad/sec from a reference rate of 1.57-25.13 rad/sec. An initial device design and prototype are presented. The device utilizes a small friction disk housed in a thimble, driven remotely from a motor worn on the user's wrist. The device directly integrates with a robotic force feedback arm, preserving the force feedback functionality while adding the tactile information on frictional sliding. Insights from the presented psychophysical experiments and initial device prototype will be utilized in the design of the next generation of rotation friction devices that will be used in multi-finger grasp studies.
\end{abstract}

\section{Introduction}

When we handle objects within our hands, rotational sliding is invariably a large component of the finger/object interaction. Pure rolling and pure translational sliding, as studied extensively in the grasping and dexterous manipulation literature, are rare. In a series of studies investigating the control of grip forces under various conditions, Johansson and his colleagues found that humans are capable of precisely and dynamically adjusting grip forces between two digits in order to maintain grasp stability de- spite changes in tangential linear forces and/or torques [8] and despite variations in the local curvatures of grasped surfaces [4]. Moreover, the adjustments in grip forces reflect the local shape and friction conditions between the contact surfaces such that a thin safety margin is guaranteed at all times. Neurophysiological evidences suggest that the rapidly adapting fibers of either the Meissner or the Pacinian mechanoreceptors are responsible for coding the relative motions between an object and the skin and for signaling pending or actual slips between the two contact surfaces [16]. Models of combined rotational and translational friction [6] also make it clear that whenever the average distance between contacts is large compared to the average radius of each contact patch, rotational sliding is likely to be more significant than translational sliding. It follows that the perception of linear and rotational slipping is an important part of manual object manipulation, and it plays an important role in our assessment of local surface texture and/or friction properties.

Realizing the limitations of point-contact stylus-based haptic interactions that are typical of most force-feedback (kinesthetic) displays, many researchers have in the recent years turned their efforts towards developing tactile displays. For example, array-based tactile displays have been used to convey local surface geometry information and to enable fingerpad/hand/palm-based haptic interaction. These include a high-density array (20-by-20 filament elements over a $10 \mathrm{~mm}-$ by- $10 \mathrm{~mm}$ area) that matches the mechanoreceptor density of the human fingerpad [13], a shape memory alloy display [5], a pneumatic tactile array display [11] and pin arrays [7, 18]. In addition, vibrotactile displays have been used effectively in teleoperation systems $[3,9,12]$. Recently, slip displays have been devel- 


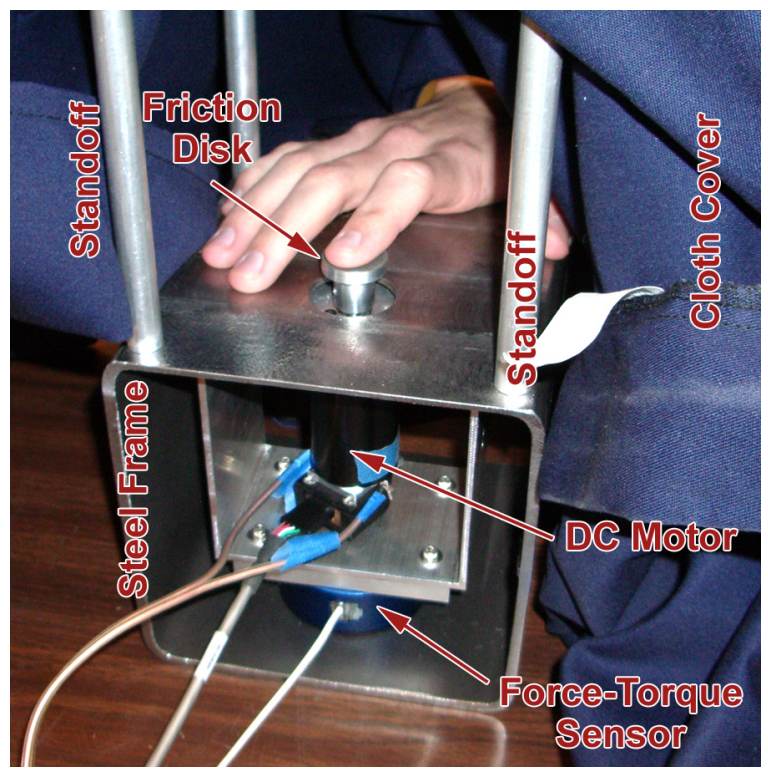

Figure 1. Experiment apparatus used in the psychophysical experiment

oped at several research laboratories to provide information about the relative motions between virtual objects and the fingertip $[15,19]$ as well as displays that render contact location or skin stretch to the fingerpad [14, 17]. New psychophysical experiments enabled by sliding displays have led to a better understanding of tactile shape perception from motion [1], human resolution of sliding speed and direction [15], and the role of slip information in grip force regulation [19].

To the best of our knowledge, the rotational friction display (RFD) presented in this article is the first attempt at displaying rotational sliding to the human fingertip. The authors of the Kinoshita et al. (1997) study commented that they were the first to study the effect of tangential torque, as opposed to tangential linear force, on the control of grip force in preventing slip [8]. However, their experimental apparatus did not allow for the precise control of rotational sliding velocity, duration or total travel. The RFD was developed specifically to simulate rotational friction and sliding and the roles they play in exploring and handling objects and tools such as flipping a screwdriver upward while holding the bottom of its handle between the thumb and index fingertips in order to reorient it for power grasping.

Understanding such ability is important step in comprehending how we perceive object rotation during 2-fingered precision grasping as explored by Goodwin [4] and Kinoshita [8]. Goodwin et al. had their subjects grasp the top of a pendulum that had a prescribed mass distribution and an instrumented handle used to measure grasp forces.

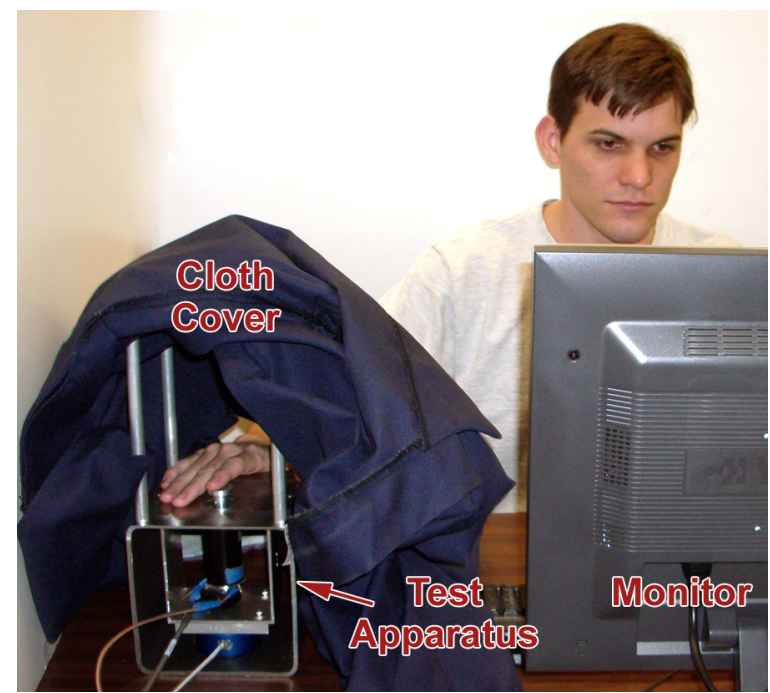

Figure 2. Experiment test setup

Their subjects tilted the pendulum $60^{\circ}$ from its resting angle and after a brief pause in the tilted position, the subjects then allowed the pendulum to rotate and descend back to its rest position ("slip" phase). In our investigation we have focused on the perceptual thresholds of rotational sliding rates (about an axis perpendicular to the fingerpad), as it seems that rates of rotation are likely to relate to the grasp force regulation required of Goodwin et al.'s [4] subjects for the controlled rotational decent of the pendulum. We are also interested in identifying the limits of perception in order to inform future device iterations of the wearable RFD and create controller performance specifications.

In the remainder of this paper, we present a detailed study of the perception of rotational friction and sliding performed on a desktop device. This study represents an important first step towards understanding the capabilities and resolution in human perception of rotational friction and sliding and lends insight into design requirements for a rotational friction display controller. Additionally, we will present a prototype design for a finger-mounted RFD that attaches to a PHANToM robotic arm to force and rotational tactile cues.

\section{Experimental Methods}

A set of experiments were completed in order to understand the human tactile discrimination threshold for perceived rotation rate of slipping at the fingertips. The details of the experiments are described below. 


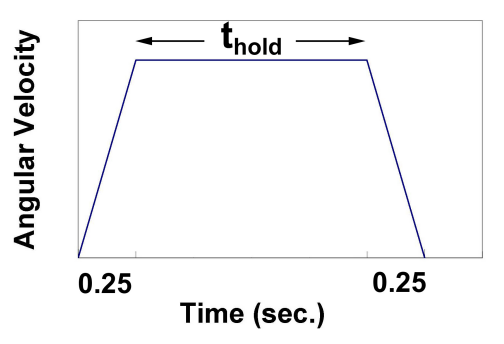

\section{Figure 3. Rotational velocity profile for rotary stimuli used in the experiments, with $t_{\text {hold }}=1$ sec.}

\subsection{Apparatus}

Figure 1 depicts the experiment apparatus used during perceptual experiments to evaluate JNDs for rotational sliding. The contact display was a small aluminum disk, roughly $2.5 \mathrm{~cm}$ in diameter, driven by a Maxon RE-35 electric motor. Surface texture was provided by 1000 grit sandpaper epoxied to the top surface of the friction disk. This treatment provided surface texture well above perceptual limits previously shown to be necessary for perceived slip [16]. The motor was mounted within an aluminum bracket affixed to a JR3 force-torque sensor (model no. 67M25-U562). An outer steel bracket encased the motor/disk setup to isolate the force-torque sensor from extraneous forces (e.g. forces exerted by subjects resting their hand on the device bracket) except for direct contact with the friction disk.

\section{$2.2 \quad$ Participants}

Eleven participants (mean age of 29.8 years, age range from 24-52 years; 10 males and 1 female) took part in this experiment. All of the participants reported normal, or corrected-to-normal, vision. By self report, 7 were righthanded and 4 were left-handed. The participants were recruited by word of mouth and did not receive any compensation for their participation.

\section{$2.3 \quad$ Stimuli}

The test stimuli were rotations of the friction disk (Fig. 1) applied to the participants' right index finger at controlled rates. Three reference rotational velocities were used: 0.25 , 1 , and $4 \mathrm{rev} / \mathrm{sec}(1.57,6.28,25.13 \mathrm{rad} . / \mathrm{sec}$.), which corresponded to typical rotational sliding rates experienced at the fingertips during slow, medium and fast object manipulation, respectively. Due to the finite bandwidth of the tactile display, the angular velocity of the rotating friction disk was ramped from a stop to each respective rotation rate over $0.25 \mathrm{sec}$., held constant for a time period of $1.0 \mathrm{sec}$., then ramped down to a stop over $0.25 \mathrm{sec}$. (see Fig. 3).

\section{$2.4 \quad$ Procedures}

A 3-interval one-up three-down adaptive procedure was used $[2,10]$. On each trial, the participant felt three rotations. Two rotations were at the reference angular velocity, $\omega_{R}$, and a third (randomly selected to be presented during the first, second or the third interval) at a target angular velocity, $\omega_{T}$, that was higher than the reference, as per standard procedure. The independent variable was the difference in the angular rate between the reference and the target (i.e., the odd interval) rotations, denoted by $\Delta \omega$. Because each of the three intervals in each trial was of equal duration, the difference between the reference and target could also be judged based on the difference in total angular rotation, denoted $\Delta \theta$; however, subjects were instructed to base their decisions on the disks rotational rate, $\Delta \omega$. Also note that especially at high reference velocities it was unlikely that subjects could track the total angular rotations over several revolutions.

Preliminary testing to determine a rough estimate of perceptual thresholds was first conducted. These results were then used to set initial target values (i.e., odd intervals) $\Delta \omega$ for each of the three reference velocities. The value of $\Delta \omega$ was increased after each incorrect response, and decreased after three consecutive correct responses. Initially, the magnitude of $\Delta \omega$ changed by $10 \%$ of $\omega_{R}$, and subsequently by $3.3 \%$ of $\omega_{R}$ after the first three reversals. A reversal occurred when $\Delta \omega$ changed from increasing to decreasing, or vice versa.

The experimental session interspersed trials from all three reference values simultaneously. Trials for each of the three reference velocities were interleaved to prevent the subject from becoming too familiar with any one reference stimulus. Each test consisted of a total of fifteen reversals, three at the initial course step size and twelve at the finer step size, per standard established procedure $[2,10]$. The JND is established as the average of the target magnitudes of the last twelve reversals. The duration of the session for a typical subject was roughly 35 minutes.

The participants were seated in front of a computer monitor in an experimental booth. The participant's right hand was placed through an opening in a cloth cover to occlude the hand from the direct view during the experiment. Standoffs on the experimental apparatus were utilized to prevent the cover from draping on the subjects hand, causing possible distractions. The participants were instructed to use their left hand to indicate the odd (target) interval by pressing the key "1", "2", or "3", on a computer keyboard, corresponding to the first, second, and third interval, respectively. 
(a)

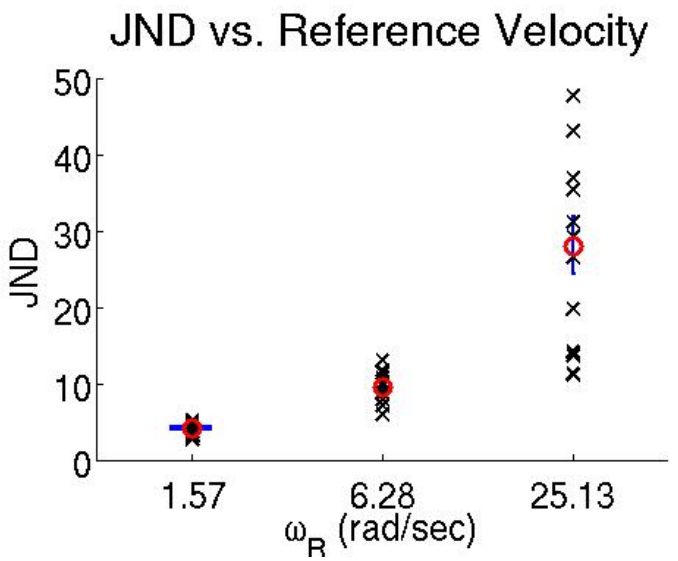

Weber Fraction vs. Reference Velocity

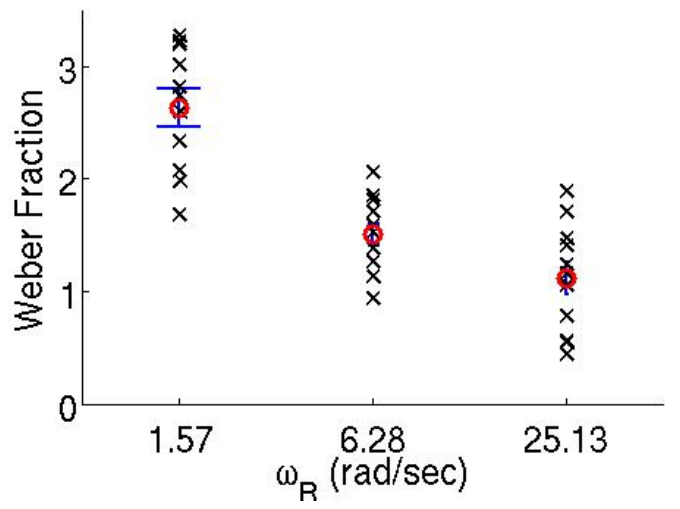

Figure 4. (a) Just-Noticeable-Difference and (b) Weber Fraction as functions of the Reference Angular Velocity. The error bars and open circles correspond to the standard error and mean for each data set

\begin{tabular}{ccccc}
\hline$\omega_{R}$ & \multicolumn{2}{c}{ JND } & \multicolumn{2}{c}{ Weber } \\
$(\mathrm{rad} / \mathrm{sec})$ & $\begin{array}{c}\text { Mean } \\
(\mathrm{rad} / \mathrm{sec})\end{array}$ & $\begin{array}{c}\text { SE } \\
(\mathrm{rad} / \mathrm{sec})\end{array}$ & Mean & SE \\
\hline 1.57 & 4.134 & 0.260 & 2.632 & 0.166 \\
6.28 & 9.442 & 0.649 & 1.503 & 0.103 \\
25.13 & 28.040 & 3.707 & 1.116 & 0.148 \\
\hline
\end{tabular}

Table 1. Experimental Results

Some participants opted to try a practice block consisting of sample trials allowing them to feel comfortable with the test procedure and exposing them to the test conditions in each test block. The participants were trained to push down on the friction disk with approximately $2 \mathrm{~N}$ of normal force before beginning the experiment. The actual normal force applied by the subjects fingertip was recorded throughout the experiment with the JR3 force/torque sensor.

\section{Results and Discussion}

Table 1 and Fig. 4 show the experimental results for all test subjects. Results for a typical test run are shown in Fig. 5. Subjects averaged between $0.56 \mathrm{~N}$ and $1.78 \mathrm{~N}$ of normal force with a standard deviation in the range of $0.12 \mathrm{~N}$ to $0.40 \mathrm{~N}$. A Weber fraction versus reference velocity plot (Fig. 4(b)), shows that the Weber fraction increases dramatically from $1 \mathrm{rev} / \mathrm{sec}(6.28 \mathrm{rad} / \mathrm{sec})$ to $0.25 \mathrm{rev} / \mathrm{sec}$ $(1.57 \mathrm{rad} / \mathrm{sec})$. The increase is much greater than between $4 \mathrm{rev} / \mathrm{sec}(25.13 \mathrm{rad} / \mathrm{sec})$ and $1 \mathrm{rev} / \mathrm{sec}$. It is very common for Weber fraction to increase sharply at the low end of stimulus intensity, as is the trend in the data shown in Fig. 4(b). This is usually interpreted as a lower limit on human resolution. In other words, JND can not decrease indefinitely
Reference Velocity $1.0 \mathrm{rev} / \mathrm{sec}(6.28 \mathrm{rad} / \mathrm{sec})$

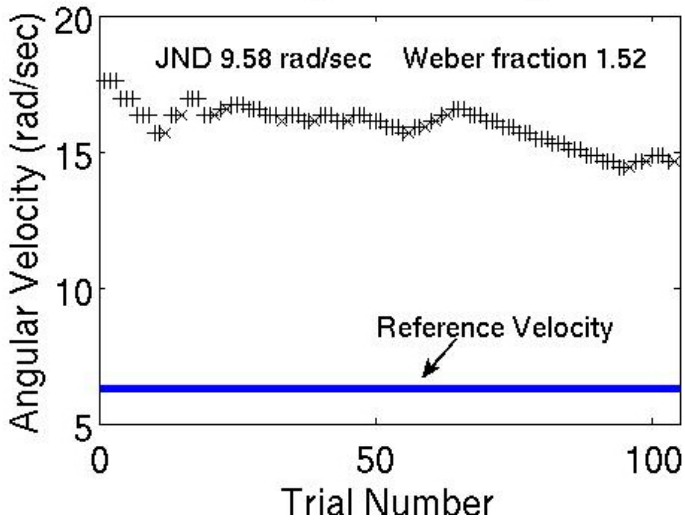

Figure 5. Typical test results as the reference decreases; JND hits a lower limit at some point. It is also possible that since slow rates of rotational sliding is quite common during in-hand manipulation tasks that humans have simply learned to ignore these signals. Or rather, the most important information during manipulation may simply be knowledge of contact location and whether or not an object is moving at a high rate (i.e., slip). Additional pilot tests performed at rotation rates higher than those presented in (Fig. 4(b)) suggest a continued decrease in the weber fraction at higher rates. However, these higher rotation rates were not included in this study as they were less relevant for manipulation. We attribute this trend to the increase in vibratory information at higher rates that would be more easily detected by the Pachinian Corpuscles. 
(a)

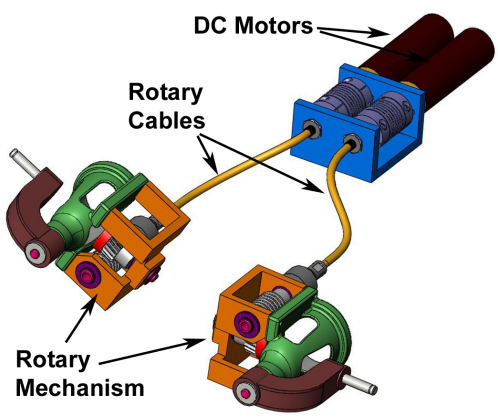

(c)

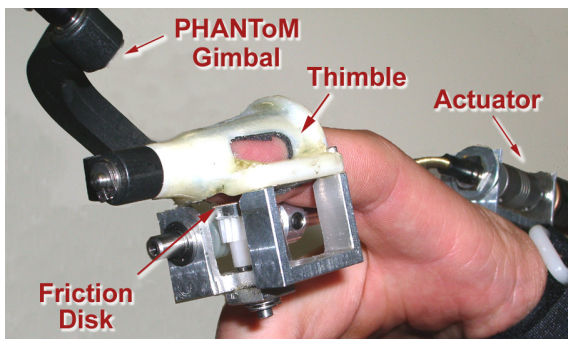

(b)

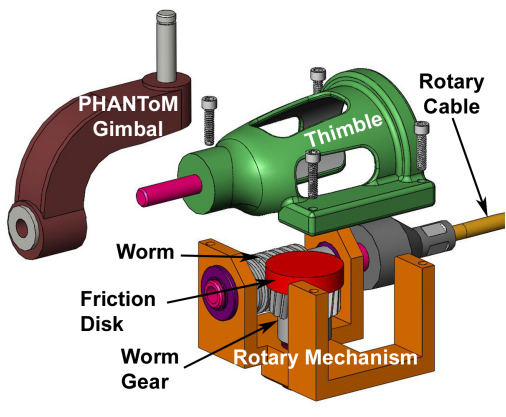

(d)

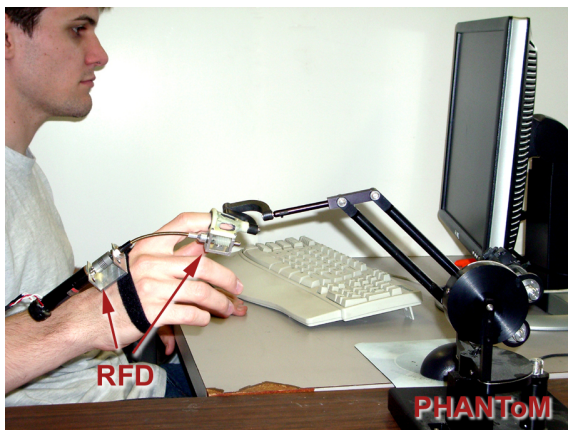

Figure 6. Rotational friction display. (a) Two devices to be used in a two-finger manipulation task. (b) Close-up view of the thimble and rotary assembly. (c) Rotational friction display mounted to a PHANTOM gimbal. (d) Rotational friction display in use

\section{Device Design}

A new tactile display was designed to be used in combination with a PHANToM robotic arm (SensAble Technologies, Inc., Woburn, MA, USA), interfacing with the last stage of the PHANToM's gimbal and replacing the provided thimble. The device utilizes a $16 \mathrm{~mm}$ friction disk placed in direct contact with the user's fingerpad. A custom-designed thimble provides an interface that is separable from the user's finger as well as to the display's rotary mechanism, as shown in Fig. 6. The thimble also provides an attachment interface to the PHANToM.

The display's drive system is divided into proximal and distal halves to reduce the inertia and package size at the (distal) fingertip. A rotary cable carries input torque from a motor secured to the dorsal side of the user's hand to the rotary mechanism that is attached to the underside of the thimble. The mechanism uses a standard nylon/acetal worm/wormgear combination to convert the rotary motion from the cable that runs along the user's finger, $90^{\circ}$ to the rotation of the disk at the user's fingerpad (see Fig. 6(b)). The axis of rotation of the disk is normal to the fingerpad. The interface between the rotary mechanism and the thimble can be shimmed so that in combination with the foam lining of the thimble a snug fit to the user's finger is ensured.

\section{Conclusions}

In this paper we present the design of a novel tactile display meant to be worn on the hand and used in combination with a PHANToM robotic force feedback device. We also present perceptual thresholds for judging rotational sliding rates at $1.57,6.28,25.13 \mathrm{rad} / \mathrm{sec}$ which corresponded to typical rotational sliding rates experienced at the fingertips during slow, medium and fast object manipulation, respectively. The JND thresholds for each of these reference rates were $4.13,9.44$, and $28.04 \mathrm{rad} / \mathrm{sec}$, respectively. We also observe that at the slowest rates tested, we are beginning to approach the limits of human perception as observed in Fig. 2.4. The data reveal that our ability to discern rotational rates of sliding is actually quite poor. This means that the performance of a rotation friction display could have modest error, and yet perform quite effectively for communicating rotational sliding information. It is suspected that the driving requirements for a device controller will be based on preserving temporal cues and minimizing possible oscillations.

Future work will include the analysis of the real-time force-torque data collected during the experiments reported here. We will also investigate the relative roles of visual and tactile cues for interpreting rotational sliding. The results from the present and planned future studies will help 
guide the revision of the RFD design with the goal to produce multiple RFD prototypes in order to conduct studies on focused grasp and multi-finger object manipulation.

\section{Acknowledgments}

The authors would like to acknowledge John Hollerbach for the use of his force sensor and Nicola Diolaiti for his efforts in helping to program the device control interface.

\section{References}

[1] A. Bicchi, D. Dente, and E. P. Scilingo. Haptic illusions induced by tactile flow. In Proceedings of EuroHaptics 2003, pages 314-329, 2003.

[2] A. Brisben, S. Hsiao, and K. Johnson. Detection of vibration transmitted through an object grasped in the hand. Journal of Neurophysiology, 81:1548-1558, 1999.

[3] J. T. Dennerlein, P. A. Millman, and R. D. Howe. Vibrotactile feedback for industrial telemanipulators. Proceedings of the Symposium on Haptic Interfaces for Virtual Environments and Teleoperator Systems, 61:189-195, 1997.

[4] A. W. Goodwin, P. Jenmalm, and R. S. Johansson. Control of grip force when tilting objects: Effect of curvature of grasped surfaces adn applied tangential torque. Journal of Neurocience, 18(24):10724-10734, 1998.

[5] C. Hasser and J. Weisenberger. Preliminary evaluation of a shape memory alloy tactile feedback display. In Proceedings of the Symposium on Haptic Interfaces for Virtual Environments and Teleoperator Systems, pages 73-80, 1993.

[6] R. Howe and M. Cutkosky. Tactile detection of slip: Surface microgeometry and peripheral neural codes. International Journal of Robotics Research, 15(6):557-72, 1996.

[7] H. Iwata, H. Yano, and R. Kawamura. Array force display for hardness distribution. Proceedings of the 10th International Symposium on Haptic Interfaces for Virtual Environment and Teleoperator Systems, pages 165-171, 2002.

[8] H. Kinoshita, L. Bäckström, J. R. Flanagan, and R. S. Johansson. Tangential torque effects on the control of grip forces when holding objects with a precision grip. Journal of Neurophysiology, 78:1619-1630, 1997.

[9] D. Kontarinis and R. Howe. Tactile display of vibratory information in teleoperation and virtual environments. Presence: Teleoperators and Virtual Environments, 4(4):387402, 1995.

[10] H. Levitt. Transformed up-down methods in psychoacoustics. Journal of the Acoustical Society of America, 49(2):467-477, 1971.

[11] G. Moy, C. Wagner, and R. S. Fearing. A compliant tactile display for teletaction. In Proceedings of the IEEE International Conference on Robotics and Automation (ICRA 'OO), volume 4, pages 3409-3415, 2000.

[12] A. Murray and R. L. K. P. Khosla. Psychophysical characterization and testbed validation of a wearable vibrotactile glove for telemanipulation. Presence: Teleoperators and Virtual Environments, 12:156-182, 2003.
[13] D. T. V. Pawluk, C. P. v. Buskirk, J. H. Killebrew, S. S. Hsiao, and K. O. Johnson. Control and pattern specification for a high density tactile array. In Proceedings of the ASME Winter Annual Meeting, Symposium on Haptic Interfaces for Virtual Environment and Teleoperator Systems, volume 64, 1998.

[14] W. R. Provancher, M. R. Cutkosky, K. J. kuchenbecker, and G. Niemeyer. Contact location display for haptic perception of curvature and object motion. International Journal of Robotics Research, 24(9):691-702, 2005.

[15] M. Salada, J. E. Colgate, P. Vishton, and E. Frankel. Two experiments on the perception of slip at the fingertip. In Proceedings of the 12th International Symposium on Haptic Interfaces for Virtual Environment and Teleoperator Systems (HAPTICS '04), pages 146-153, 2004.

[16] M. A. Srinivasan, J. M. Whitehouse, and R. H. LaMott. Tactile detection of slip: Surface microgeometry and peripheral neural codes. Journal of Neurophysiology, 63(6):13231332, 1990.

[17] N. D. Sylvester and W. R. Provancher. Effects of longitudinal skin stretch on the perception of friction. To be Presented at the Second Joint Eurohaptics Conference and Symposium on Haptic Interfaces for Virtual Environment and Teleoperator Systems, 2007.

[18] C. R. Wagner, S. J. Lederman, and R. D. Howe. Design and performance of a tactile shape display using rc servomotors (short paper). Haptics-e: The Electronic Journal for Haptics Research, 3(4):www.haptics-e.org, 2004.

[19] R. J. Webster, T. E. Murphy, L. N. Verner, and A. M. Okamura. A novel two-dimensional tactile slip display: design, kinematics and perceptual experiments. ACM Transactions on Applied Perception, 2(2):150 - 165, 2005. 